corresponding to minimum computed energy are not necessarily the same for a bond in a dipeptide as for the same bond in the nonapeptide, for example. Ponnuswamy et al. assert that at a chain length of nine the conformation begins to home in on the true geometry, so that long-range interactions between remote parts of the chain need often not be considered. The results, however, show that there is still a long way to go. The unpalatable fact remains that the experimental conformation of excised peptide segments is all but unrelated to their state in the native protein, so that failure to take account of long-range interactions is by no means a trivial limitation.

Crawford, Lipscomb and Schellman (ibid., 538) have concentrated on the hairpin turn of the polypeptide chain as a structural landmark suitable for purposes of prediction. This turn is defined by four residues, and Crawford et al. convey the surprising information that no less than 33 per cent of the residues of the globular proteins in their sample are engaged in such formations, as against 34 per cent in helices and 17 per cent in pleated sheets. Asparagine, glycine and proline have a strong tendency to appear in the reverse turns. They are most often in the first, second and third position respectively, and tryptophan is frequently found in the fourth. A case for a special function for leucine residues is made by Chou and Fasman (J. Mol. Biol., 74, 263; 1973), who give experimental evidence that this residue confers the greatest stability on polypeptide $\alpha$-helices. Its occurrence is most frequent in internal helices in globular proteins, and around the walls of crevices containing prosthetic groups, and Chou and Fasman suggest that it may have an important structure nucleation function.

On the nucleation of chain folding, Wetlaufer (Proc. US Nat. Acad. Sci., 70, 697 ; 1973) has found on examining the structures of many globular proteins that spatially separate compact folded regions are often present, by criteria which he defines, and he gives arguments why it might be anticipated that chain folding would ensue after the formation of independent conformational nuclei in each separate domain.

Some potential energy calculations on $\beta$-pleated sheets have been done by Chothia (J. Mol. Biol., 75, 295 ; 1973), who finds that a right-handed twist in the direction of the polypeptide chain, which corresponds to dihedral angles within one zone of the conformational map, makes for minimization of energy. This sense of twist turns out to prevail in $\beta$-structures in known globular proteins. A structure with a left-handed twist would have appreciably greater conformational freedom, with relatively large variability of dihedral angles.
PALAEOMAGNETISM

\section{Dafing Hadrian's Wall}

from our Geomagnetism Correspondent

THE success of palaeomagnetism rests on the fortunate fact that many rocks contain magnetic grains which have very high coercive forces. When a rock forms, its magnetic components acquire a magnetization in the direction of the ambient geomagnetic field; and because many of the grains have high coercive forces, the magnetization becomes "locked in" to the rock, usually at temperatures between $500^{\circ}$ and $700^{\circ} \mathrm{C}$, and is thereafter largely unsusceptible to the vicissitudes of subsequent history. Accordingly, many rocks still contain a valid record of the direction of the geomagnetic field at the time of their formation, perhaps thousands of millions of years ago. The palaeomagnetist simply has to measure such directions to determine the properties of the Earth's magnetic field in the past.

Unfortunately, this task is frequently complicated by the fact that all grains do not have high coercive forces. The magnetic particles in any given rock sample are likely to cover a range of compositions, sizes and physical structures - all factors which determine coercive force - and thus a range of coercive forces. Grains throughout the whole of this range will acquire magnetizations in the direction of the original field; but grains with lower coercive forces will be less inclined to maintain the direction throughout time in the face of random temperature fluctuations. Thus if the orientation of the rock with respect to the external field changes (for example, because of continental drift or because of field reverses), the grains with lower coercive forces may, and often do, lose their original magnetization and acquire a new one in the new field direction. In short, they are magnetically unstable and thus subject to viscous remagnetization which can take place in times ranging from seconds to hundreds of thousands of years.

To most palaeomagnetists, viscous magnetization is a nuisance to be removed (usually by thermal or alternating field demagnetization) before the more permanent record of the ancient magnetic field can be measured, although there have been numerous investigations of viscous magnetization as a phenomenon in its own right. Heller and Markert (Geophys. J., 31, 395 ; 1973) have, however, now attempted to put viscous magnetization to practical use in the dating of rock movements. The theoretical basis of their work is chiefly a series of investigations carried out by Néel (see, for example, Phil. Mag. Suppl. Adv. Phys., 4, 191 ; 1955) during the late 1940s and early 1950s. This theory is complicated, incomplete and probably defective in part, although with some modification and addition it has proved sufficient for the present purpose. Using the theory as a basis, Heller and Markert have developed a set of experimental procedures involving alternating field demagnetization to determine the time at which viscous magnetization began to form in any given rock.

The rocks chosen by Heller and Markert to test the method were quartz dolerites built into Hadrian's Wall near Greenhead in Northumberland, and they almost certainly originated from

\title{
Singularities and the C Field
}

Abour ten years ago Hoyle and Narlikar developed and published a model of the creation of matter which depends on a small and elegant modification of the field equations of general relativity. The modification was called the " $C$ " field, a negative energy field whose gradient is not a conserved flux but has sources where matter is created. The motivation for this work was the steady-state cosmology according to which, in spite of the expansion of the Universe, the density of matter remains everywhere constant in time.

It is a measure of the rate of progress of observational cosmology that the original motivation for this work has largely disappeared; there are few cosmologists who now take seriously the model of the Universe which looks the same in every direction in every place at every time. But the need to modify the equations of general relativity remains, because the usual equations possess no solutions free from singulari- ties. There is not even a model of a massive object collapsing under its own weight without an elastic repulsion. Penrose and Hawking and others have shown that singularities occur under quite wide and physically reasonable conditions. Several workers are seeking a quantization of the equations to avoid the singularity.

In next Monday's Nature Physical Science (April 30) Narlikar now points out that the classical modification made for the steady-state theory can also avoid the singularity. He presents the equations in a new way and works out two special cases. One is the steady-state theory with a constant matter density. The other is the big-bang model modified by the fact that the creation of matter at the origin is at the expense of the negative energy $C$ field. The resulting geometrical structure is similar to that of a model with torsion put forward by Trautman (Nature Physical Science, 242, 7 ; 1973). 